inhibitors on their effects on $\mathrm{T}$ cells. As these differences remained even after controlling for potency, this suggests the specificity profiles toward individual HDACs was responsible for their unique effects.

Conclusions These studies demonstrate clear differences in the effect of HDACi on cytokine production by distinct $\mathrm{T}$ cell subsets. Ongoing studies are aimed at elucidating the specific HDACs responsible for regulating $\mathrm{T}$ cell effector functions and tumor immunogenicity when targeted. Ultimately, understanding this could help identify inhibitors with the desired specificity profile for combining with immunotherapy.

http://dx.doi.org/10.1136/jitc-2020-SITC2020.0821

\section{LOCAL RADIOTHERAPY SYNERGIZES WITH TUMOR- SPECIFIC TCR REDIRECTED T CELLS IN THE REJECTION OF PROSTATE CANCER}

${ }^{1}$ Marco Catucci* ${ }^{*}$ Veronica Basso, ${ }^{1}$ Chiara Lucrezia Deantoni, ${ }^{1}$ Simone Baroni, ${ }^{1}$ Antonello Spinelli, 'Massimo Freschi, ${ }^{1}$ Cesare Cozzarini, ${ }^{1}$ Claudio Fiorino, ${ }^{1}$ Nadia Di Muzio ${ }^{2}$ Silvia Formenti, ${ }^{2}$ Sandra Demaria, ${ }^{1}$ Anna Mondino. ${ }^{1}$ IRCCS San Raffaele Scientific Institute, Milan, Italy; ${ }^{2}$ Weill Cornell Medicine, New York, NY, USA

Background Adoptive $\mathrm{T}$ cell therapy (ACT) has become a promising option for cancer patients. While tumor-infiltrating lymphocytes were initially exploited as a source of tumor reactive lymphocytes, $\mathrm{T}$ cells genetically redirected to the tumor by TCR/CAR gene transfer are now in clinical validation. In the case of solid tumors, unfavorable immunosuppressive microenvironments remain recognized barriers to therapeutic efficacy. We have recently reported that the therapeutic activity of ACT against poorly immunogenic and indolent prostate cancer is improved by the concurrent targeting of the tumor stroma by mean of $\mathrm{T}$ cells redirected to an ubiquitously expressed minor histocompatibility antigen or a tumor vessel targeted TNF derivative. We have now taken the concept further and hypothesized that local radiotherapy (RT), might also synergize with ACT by promoting lymphocyte endothelial transmigration and tumor recognition, and ultimately favor abscopal effects.

Methods We investigated the combination of local RT and ACT in TRAMP (Transgenic Adenocarcinoma of the Mouse Prostate) mice and in mice bearing subcutaneous B16/B16OVA (MO4) or TRAMP-C2/TRAMP-C2-OVA tumors. Local RT was delivered by X-RAD SmART (the Small Animal Radiation Therapy) microirradiator in single dose or hypo-fractioned regimens. ACT consisted of $\mathrm{T}$ cells engineered with tumor-specific TCRs. Immunogenic consequences were analyzed by Real-Time PCR, and flow cytometry (FACS) analyses. Prostate tumor debulking was evaluated by histological analyses.

Results We found that local hypofractionated RT and ACT, while individually inefficacious in controlling tumor growth, concurred to the debulking of advanced prostate adenocarcinoma when used in combination in treating TRAMP mice. Mechanistically, exposing isolated tumor cells, or the TRAMP mouse prostate to hypo-fractionated RT regimens induced stronger type-I interferon (IFN-I) responses, when compared to single high dose. Acutely, hypofractionated RT promoted better immune tumor infiltration, among which TCR redirected effector cells.

Conclusions Data support feasibility and efficacy of combining hypo-fractionated local RT with ACT in the form of TCR engineered $\mathrm{T}$ cells to promote prostate cancer recognition and eradication. Tumor debulking was observed in the absence of treatment-related toxicity. Systemic recirculation of TCR redirected $\mathrm{T}$ cells was observed. We are now investigating therapeutic effects at distal (metastatic) sites.

Acknowledgements The authors acknowledge the support of the Italian Association for Cancer Research (AIRC)

Ethics Approval The studies involving animals were approved by The Institutional Ethical Committee (IACUC\#999).

http://dx.doi.org/10.1136/jitc-2020-SITC2020.0822

\section{CD4 T CELLS ARE ESSENTIAL FOR AN ANTI-TUMOR EFFECT IN A B78 MURINE MELANOMA TUMOR MODEL}

Arika Feils*, Mackenzie Heck, Anna Hoefges, Peter Carlson, Luke Zangl, Ravi Patel, Dmitriy Kravtsov, Jacquelyn Hank, Zachary Morris, Alexander Rakhmilevich, Paul Sondel, Amy Erbe. University of Wisconsin-Madison, Fitchburg, WI, USA

Background Mice bearing B78 melanoma tumors can be cured using an in situ vaccine (ISV) regimen that includes radiation (RT) together with immunocytokine (tumor-targeting mAb conjugated to IL-2). B78 melanoma cells, derived from B16 cells, express minimal to no MHC-I but express MHC-II upon IFNg/TNF-a stimulation. Although B78 cells are primarily MHC-Ideficient, an increased CD8 $\mathrm{T}$ cell infiltration into the tumor microenvironment (TME) has been shown following ISV. ${ }^{1}$ To further investigate the potential role of specific immune cell lineages in the B78 anti-tumor response to ISV, immune subset depletion studies and flow cytometric analyses were performed. Methods C57BL/6 mice bearing B78 tumors were depleted of immune cell subsets with mAbs (anti-CD4, anti-CD8, antiNK1.1, or Rat IgG control) for 3 weeks during the course of treatment. Treatment groups included no treatment, RT (12 Gy), or ISV (RT D0 and immunocytokine D5-D9). 6 mice/ group (repeated three times) were followed for survival/tumor growth, and flow cytometry studies included 4 mice/group, sacrificed on D8 and D13 following the start of ISV.

Results Mice depleted of CD4 T cells during the course of ISV showed a significant reduction of anti-tumor effect as compared to mice treated with ISV/Rat IgG ( $p$

Conclusions These studies suggest that CD4 T cells are essential for an anti-tumor response in the B78 melanoma model. In vivo depletion data show that CD4 $\mathrm{T}$ cells, but not CD8 or NK cells, are required for a decrease in tumor growth via ISV. Flow cytometric analyses suggest an interplay between CD4 and CD8 T cells as indicated by a decrease in CD8/IFN$\mathrm{g}$ expression following ISV in the absence of CD4 $\mathrm{T}$ cells. The role that MHC-I and MHC-II expression plays in this CD4/CD8 $\mathrm{T}$ cell anti-tumor response is under investigation. In future studies, B78 melanoma may serve as a critical syngeneic model for development of more effective immunotherapy treatment regimens.

Ethics Approval All animal experiments were performed in accordance with protocols approved by Animal Care and Use Committees of the University of Wisconsin-Madison.

\section{REFERENCE}

1. Morris Z, Guy E, Francis D, et al. In situ tumor vaccination by combining local radiation and tumor-specific antibody or immunocytokine treatments. Cancer Res 2016;76(13):3929-3941

http://dx.doi.org/10.1136/jitc-2020-SITC2020.0823 